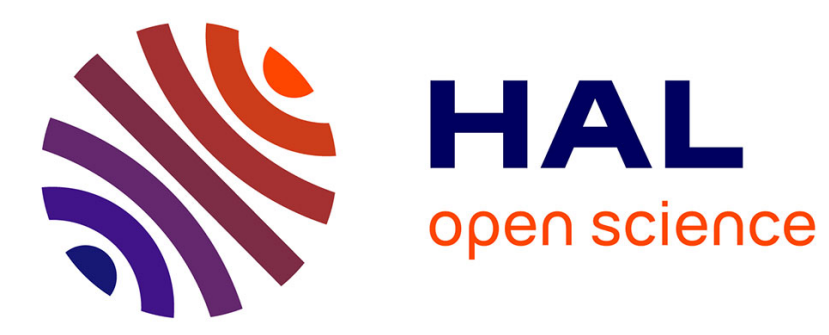

\title{
Measure of Straight Lines for Digital Contour Analysis
}

David Coeurjolly, Isabelle Sivignon

\section{To cite this version:}

David Coeurjolly, Isabelle Sivignon. Measure of Straight Lines for Digital Contour Analysis. International Journal of Imaging Systems and Technology, 2011, pp.67-75. 10.1002/ima.20268 . hal00529817

\section{HAL Id: hal-00529817 \\ https://hal.science/hal-00529817}

Submitted on 26 Oct 2010

HAL is a multi-disciplinary open access archive for the deposit and dissemination of scientific research documents, whether they are published or not. The documents may come from teaching and research institutions in France or abroad, or from public or private research centers.
L'archive ouverte pluridisciplinaire HAL, est destinée au dépôt et à la diffusion de documents scientifiques de niveau recherche, publiés ou non, émanant des établissements d'enseignement et de recherche français ou étrangers, des laboratoires publics ou privés. 


\title{
Measure of Straight Lines for Digital Contour Analysis ${ }^{\star}$
}

\author{
David Coeurjolly and Isabelle Sivignon \\ Université de Lyon, CNRS, LIRIS, UMR5205, F-69622, France \\ \{david.coeurjolly, isabelle.sivignon\}@liris.cnrs.fr
}

\begin{abstract}
In digital geometry, objects such as digital straight lines can be considered as equivalent classes of Euclidean straight lines with respect to a digitization process. This paper investigates the analysis of the set of straight lines whose digitization is a given digital straight segment with the help of tools from integral geometry. After a definition of a measure on such sets, we illustrate several applications of it in digital geometry for contour analysis.
\end{abstract}

\section{Introduction}

When investigating relationships between Euclidean and Digital geometries, we are facing difficulties induced by the digitization process: Given a digital set $X \subset \mathbb{Z}^{2}$, there is an infinity of shapes $\Omega_{X} \subset \mathbb{R}^{2}$ such that $X=\Omega_{X} \cap \mathbb{Z}^{2}$ (in this statement, we have considered the Gauss digitization process (Klette and Rosenfeld, 2004b) for the sake of simplicity). The set $\Omega_{X}$ can be considered as the preimage of $X$ for the Gauss digitization. If we want to perform geometrical analysis on digital objects we usually associate properties on $X$ from properties of its preimage set $\Omega_{X}$. For example, $X$ is digitally convex if there exists a continuous convex shape in $\Omega_{X}$ (Sklansky, 1972). In shape analysis, many approaches exist to decide if the set $X$ is digitally straight (see Klette and Rosenfeld 2004a for a survey). Among all the definitions of Digital Straight Lines (DSL for short), we consider the following one: $X$ is a DSL if there exist a Euclidean straight line in $\Omega_{X}$.

In this paper we focus on the analysis of a subset of $\Omega_{X}$ which only corresponds to straight lines: we define $\mathcal{L}_{X}$ the set of straight lines (maybe empty) such that the digitization of each line in $\mathcal{L}_{X}$ is exactly $X$. Note that we can define a digital straight segment (DSS for short) and its associated preimage as the set of Euclidean lines whose digitization contains $X$. In the literature, the preimage $\mathcal{L}_{X}$ has been widely used to control DSS recognition algorithms (Dorst and Smeulders, 1984, 1991; Lindenbaum and Bruckstein, 1993; Klette and Rosenfeld, 2004a), or to perform geometric reconstruction from a digital contour (Breton et al., 2003). In some sense, $\mathcal{L}_{X}$ can be considered as an uncertainty characterization of $X$ with straight lines: Given a set $X$, the thinner the $\mathcal{L}_{X}$, the finer the DSS characterization of $X$.

* This work was partially funded by the ANR project GeoDIB ANR-06-BLAN-0225. 
We propose here to formalize and investigate this preimage analysis with the help of integral geometry results (Santaló, 1985). More precisely, we use the mathematical measure of lines in the plane to evaluate a kind of accuracy of the DSS recognition. Similar analyses have been proposed in (Kiryati et al., 1991) to compare the continuous and the analytical Hough transforms. The authors have considered the measure of the DSS worst case in both models for recognition purposes. In the framework of line detection, other approaches have been developed to estimate the distortion of the line detected compared to the ideal line (Caglioti, 1993). In this paper, we first provide a closed formula to evaluate the measure of lines in Section 2 before studying it in the special case of digital straight segment in Section 3. Finally, we illustrate several applications in digital geometry for contour analysis in Section 4 .

\section{Measure of Straight Lines in the Plane}

\subsection{Preliminaries}

In this section, we first recall integral geometry results (Santaló, 1985). First of all, let us consider a polar representation $(\rho, \phi)$ of a straight line $l$ with $\phi \in[0,2 \pi]$ and $\rho \geq 0$. Hence, a point $p(x, y) \in \mathbb{R}^{2}$ belongs to $l$ if

$$
x \cos \phi+y \sin \phi-\rho=0 .
$$

In integral geometry and in the theory of geometrical probability, the measure of a set $\mathcal{L}$ of lines is defined as the integral over the set of a differential form $\omega=f(\rho, \phi) d \rho \wedge d \phi$, provided the fact that this integral exists in the Lebesgue sense. The differential form is defined using notations and rules of the exterior calculus for which an overview is given in (Santaló, 1985). In our context, exterior calculus will coincide with classical differential calculus in most situations.

To define a measure of straight lines, the function $f$ should be chosen such that the resulting integral is invariant under the group of motions in the plane (rotation and translation). In fact, the invariance implies that $f$ is constant (Crofton, 1868; Santaló, 1985). Hence using $f(\rho, \phi)=1$ we have:

Theorem 1 ((Santaló, 1985)). The measure of a set of lines $G(\rho, \phi)$ is defined by the integral over $G$ of the differential form

$$
d G=d \rho \wedge d \phi .
$$

Note that the measure is always taken at absolute value. In the following, since we will only consider continuous and closed domains $G$, the integral always exists in the Lebesgue sense. As a consequence, the measure is positive, monotonic (if $A \subseteq B$, then $m(A) \leq m(B)$ ), additive (if $A \cap B=\emptyset$, then $m(A \cup B)=$ $m(A)+m(B))$, and the measure of the empty set is 0 . 


\subsection{Density in the $(a, b)$-linear space}

As defined above, the measure definition perfectly matches with the $(\rho, \phi)-$ Hough transform for computer vision purposes (Duda and Hart, 1972). Indeed, given a continuous and closed domain $G$ in the $(\rho, \phi)$ space, the measure of $G$ is

$$
m(G)=\int_{G} d \rho \wedge d \phi,
$$

which simply corresponds to the area of $G$ in $(\rho, \phi)$.

In digital geometry, when considering DSS preimages (Dorst and Smeulders, 1984; McIlroy, 1985; Dorst and Smeulders, 1991; Lindenbaum and Bruckstein, 1993; Klette and Rosenfeld, 2004a), we usually prefer the slope-intercept linear parameter space, denoted $(a, b)$-space in the following, since it allows to characterize arithmetical properties embedded in DSS. This parameter space maps the line $a x-y+b=0$ to the point $(a, b)$. Conversely, straight lines in the $(a, b)-$ space are mapped onto points in the primal space.

In order to obtain the measure of straight lines in this space, we need to perform a transformation in the integral calculus. From (Santaló, 1985), we know that if the straight lines are parametrized by their intercept $u$ (resp. $v$ ) along the $x$-axis (resp. $y$-axis), we can change the coordinates

$$
\left\{\begin{array}{l}
\rho=\frac{u v}{\sqrt{u^{2}+v^{2}}} \\
\phi=\arctan (u / v)
\end{array}\right.
$$

to obtain

$$
d G=\frac{u v}{\left(u^{2}+v^{2}\right)^{\frac{3}{2}}} d u \wedge d v .
$$

If we consider now the $(a, b)$ parametrization widely used in digital geometry, we define the transformation:

$$
\left\{\begin{array}{l}
u=-\frac{b}{a} \\
v=b
\end{array}\right.
$$

and obtain

$$
\left\{\begin{array}{l}
\rho=\frac{b}{\sqrt{1+a^{2}}} \\
\phi=\arctan \left(-\frac{1}{a}\right)
\end{array}\right.
$$

whose Jacobian matrix is

$$
J=\left[\begin{array}{cc}
\frac{a b}{\left(1+a^{2}\right)^{3 / 2}} & \frac{1}{\sqrt{1+a^{2}}} \\
\frac{1}{1+a^{2}} & 0
\end{array}\right]
$$

Hence,

$$
d G=d \rho \wedge d \phi=|\operatorname{det}(J)| d a \wedge d b
$$

Finally,

Lemma 1. The measure of a set of lines $G(a, b)$ is defined by the integral over $G$ of the differential form

$$
d G=\frac{d a \wedge d b}{\left(1+a^{2}\right)^{\frac{3}{2}}} .
$$


Even if the $(a, b)$-parametrization leads to a more complex differential form, we demonstrate in the next sections that the measure of DSS preimages in the $(a, b)$-space can be easily computed. The Fig. 1 represents the density function as a surface plot in the $(a, b)$-parameter space.

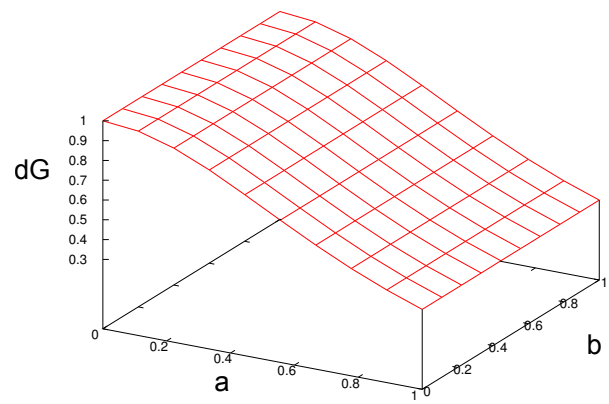

Fig. 1. Density of straight lines in the $(a, b)$-parameter space.

In terms of geometric probability, the measure allows us to obtain interesting properties. For example given two bounded and closed domains $\Omega$ and $\Omega^{\prime}$ with $\Omega^{\prime} \subset \Omega$ and $\Omega, \Omega^{\prime} \subset \mathbb{R}^{2}$, the probability that a random line intersects $\Omega^{\prime}$ with the hypothesis that it also intersects $\Omega$ is $p=\frac{m\left(\Omega^{\prime}\right)}{m(\Omega)}$, i.e. the ratio of the measure of the two domains, as defined in Equ. (2). For further developments on probability results from a measure, we invite the reader to refer to (Santaló, 1985).

\subsection{Measure of Lines of a closed $(a, b)$-space domain}

Let us consider a polygonal domain $P$ in the $(a, b)$-space defined by its boundary vertices $\left\{p_{i}=\left(a_{i}, b_{i}\right)\right\}_{i=0 . . n-1}$ (counter-clockwise). Given a function $f$ measurable on $P$ (in the Lebesgue sense), a classical way to compute the integral of $f$ over $P$ is to decompose the computation into a finite summation of triangular integration processes. As illustrated in Fig. 2, let $T_{i}$ be the oriented triangle $\left\{(0,0), p_{i}, p_{(i+1 \bmod n)}\right\}$ for $0 \leq i \leq n-1$. For each triangle $T_{i}$, let $\delta_{i}$ be the sign of its signed area $\left(\delta_{i}=\operatorname{sign}\left(a_{i} b_{i+1}-a_{i+1} b_{i}\right)\right)$.

In the rest of the paper, all index shifts such as $i+j$ must be considered modulo $n$. Using the above notations

$$
\int_{P} f(a, b) d a d b=\sum_{i=0}^{n-1} \delta_{i} \int_{T_{i}} f(a, b) d a d b .
$$

The next step is to compute the integration over triangles $T_{i}$. To solve that problem, we also consider a linear variable transformation which maps the triangle $T_{i}$ to the unit triangle $\{(0,0),(1,0),(0,1)\}$

$$
\left\{\begin{array}{l}
a=a_{i} a^{\prime}+a_{i+1} b^{\prime} \\
b=b_{i} a^{\prime}+b_{i+1} b^{\prime}
\end{array} \quad \text { whose Jacobian matrix is } J_{i}=\left[\begin{array}{ll}
a_{i} & a_{i+1} \\
b_{i} & b_{i+1}
\end{array}\right]\right.
$$




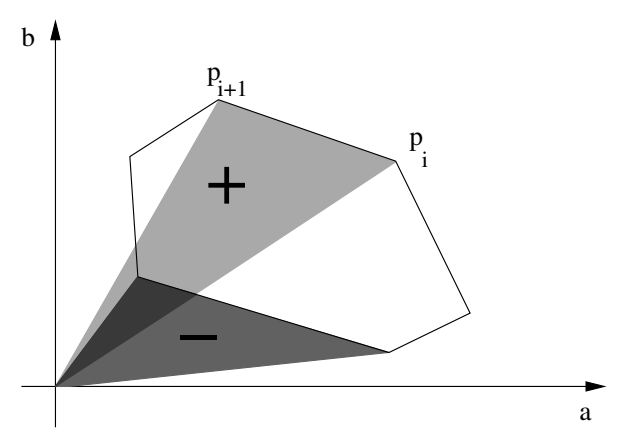

Fig. 2. Integration scheme over a polygonal domain.

Thus

$$
\int_{T_{i}} f(a, b) d a d b=\left|\operatorname{det}\left(J_{i}\right)\right| \int_{0}^{1} \int_{0}^{1-b^{\prime}} f\left(a^{\prime}, b^{\prime}\right) d a^{\prime} d b^{\prime}
$$

Note that the sign of $\operatorname{det}\left(J_{i}\right)$ and $\delta_{i}$ coincides. Finally, we obtain the following lemma:

Lemma 2. The measure of a set of straight lines defined in a polygonal domain $P=\left\{\left(a_{i}, b_{i}\right)\right\}_{i=0 . . n-1}$ in the $(a, b)$-space is

$$
m(P)=\sum_{i=0}^{n-1}\left(a_{i} b_{i+1}-a_{i+1} b_{i}\right) \cdot g\left(a_{i}, a_{i+1}\right)
$$

with

$$
g\left(a_{i}, a_{i+1}\right)= \begin{cases}\frac{1}{1+a_{i}^{2}+\sqrt{1+a_{i}^{2}}} & \text { if } a_{i}=a_{i+1}, \\ \frac{1}{1+\sqrt{1+a_{i}^{2}}} & \text { if } a_{i+1}=0 \\ \frac{1}{1+\sqrt{1+a_{i+1}^{2}}} & \text { if } a_{i}=0 \\ \frac{a_{i+1}-\sqrt{1+a_{i}^{2}} a_{i+1}+a_{i}\left(-1+\sqrt{1+a_{i+1}^{2}}\right)}{a_{i} a_{i+1}\left(a_{i+1}-a_{i}\right)} & \text { otherwise. }\end{cases}
$$

Proof. With Eq. (9), (10) and (12), we have

$$
m(P)=\sum_{i=0}^{n-1}\left(a_{i} b_{i+1}-a_{i+1} b_{i}\right) \int_{0}^{1} \int_{0}^{1-b^{\prime}} \frac{d a^{\prime} d b^{\prime}}{\left(1+\left(a_{i} a^{\prime}+a_{i+1} b^{\prime}\right)^{2}\right)^{\frac{3}{2}}}
$$

Then, thanks to the mapping of $T_{i}$ triangles to the unit triangle, the rightmost integral term can be evaluated to obtain closed formulas presented in Eq. (14). The function $g\left(a_{i}, a_{i+1}\right)$ in the lemma statement defines the result of the integral computation obtained by a symbolic mathematical solver. 


\section{Measure of Digital Straight Segments}

\subsection{DSS Preimage and Farey Fan}

In the following, we use the preimage definition proposed by Lindenbaum and Bruckstein (1993). Consider a straight line $y=a_{0} x+b_{0}$ (without loss of generality, $\left(a_{0}, b_{0}\right) \in[0,1] \times[0,1]$, i.e. straight lines are supposed to be in the first octant), the digitization of this line using the Object Boundary Quantization (see (Jonas and Kiryati, 1997) for a survey on digitization schemes) on a unit grid is the set of discrete points such that $X=\left\{(x, y) \in \mathbb{Z}^{2} \mid\left\lfloor a_{0} x+b_{0}-y\right\rfloor=0\right\}$. The preimage of $X$ is thus defined by

$$
\mathcal{L}_{X}=\{(a, b) \in[0,1] \times[0,1[\mid \forall(x, y) \in X, y \leq a x+b<y+1\} .
$$

In the $(a, b)$-space $\mathcal{L}_{X}$ is a convex polygon whose vertices are embedded in a strong arithmetical structure, the Farey Fan (McIlroy, 1985). Indeed, each pixel in $X$ induces two linear constraints with integer coefficients. The Farey Fan of order $n$ is the arrangement obtained as the intersection of all possible linear constraints associated to pixels in $[0, n] \times[0, n]$. In other words, it is the arrangement of the set of lines $\{y / x, 0 \leq y \leq x, 1 \leq x \leq n\}$ in the domain $[0,1] \times[0,1]($ McIlroy, 1985). The vertex coordinates of this arrangement are irreducible fractions whose denominator is less or equal to $n$. Finally, such an arithmetical structure allows us to design fast recognition algorithms since the cells in the Farey Fan of order $n$ defines all DSS of length $n+1$ in the first octant(Dorst and Smeulders, 1991; Lindenbaum and Bruckstein, 1993).

A first application of the measure is to merge both arithmetical properties and uncertainty within the same framework. Indeed, each cell of a Farey Fan of order $n$ represents a DSS $X$ of length $n+1$. With the measure associated to this preimage (and thus to $X$ ), we can evaluate the quantity (in terms of integral measure) of straight lines whose digitization contains $X$. Hence, Fig. 3 illustrates Farey Fans of order from 0 to 5 in which the gray value corresponds to the measure computed with Lemma 2 on each cell (the white color cell is the preimage with maximal measure). As expected, we can observe that the cells have a high variability with respect to the measure. If we consider DSS examples in Fig. 4, the first two DSS preimages are in the Farey Fan of order 4 (Fig. 3- $(e)$ ). However, if we consider the following probability test: given a random straight line $l$ whose slope and intercept is in $[0,1] \times[0,1]$, the probability such that the digitization of $l$ contains the left DSS is 3.7 times higher than the probability of the DSS in the middle. Hence, the measure is a powerful tool to perform $a$ posteriori evaluation of DSS recognitions.

Now let us investigate the relation between the measure and other DSS geometric features like DSS length or the area of DSS preimages. Consider for instance the DSS of Fig. 4. The first two DSS are of length 5, while the third one is of length 6 . However, the measure associated to the DSS of length 6 is higher than the measures of the DSS of length 5 . Thus, it seems that the relation between DSS length and DSS preimage measure is not straightforward, and we deepen the analysis hereafter. 


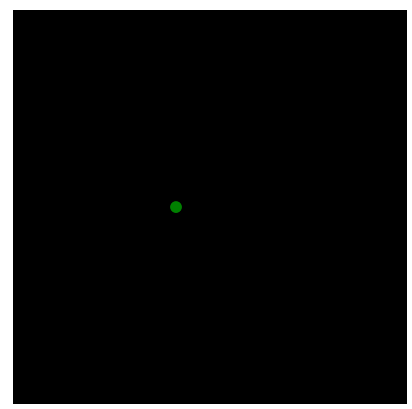

(a)

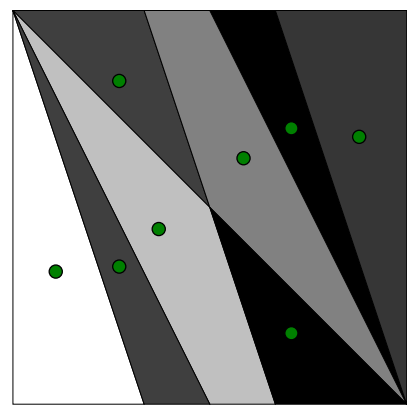

(d)

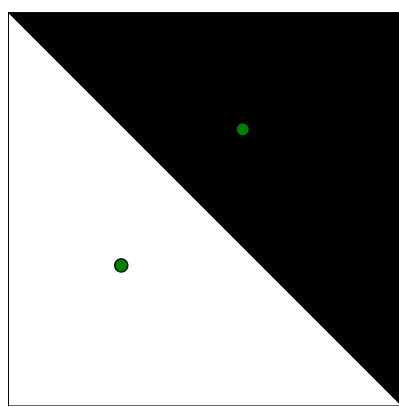

(b)

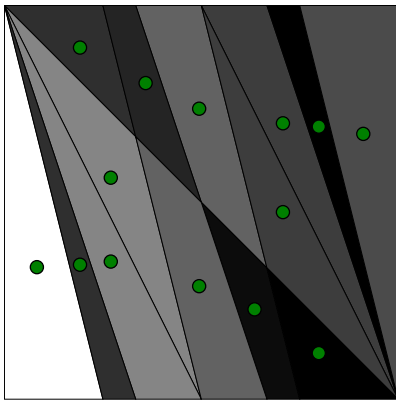

(e)

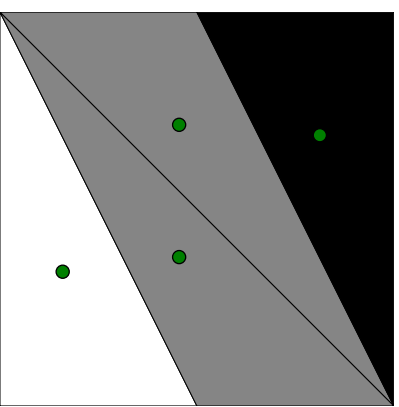

(c)

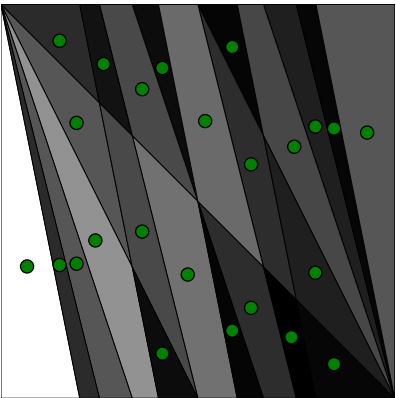

(f)

Fig. 3. Farey Fan of order from 0 to $5((a)-(f))$ with the center of mass for each preimage. The color corresponds to a linear mapping between the preimage measure and the gray level map (the white color cell is the preimage with maximal measure).

To do so, for each Farey Fan of order between 0 to $n$ (with $n=40$ in the experiments), the measure and the area of each cell of the Farey Fan is computed. Since a Farey Fan of order $l$ represents all the DSS of length $l+1$, an exhaustive study of the relations between the geometric features is performed. In Fig. 5 (a), the preimage measure is plotted against the DSS length in logarithmic scale. This shows that the measure is generally not correlated to the DSS length. More precisely, from the red and blue curves plotted on this figure, we can tell that, if $l$ stands for the DSS length, the highest measure of DSS is some $\Omega(1 / l)$ while the lowest measure is some $O\left(1 / l^{3.6}\right)$.

In Fig. 5 (b), the preimage measure is plotted against the preimage area in logarithmic scale for all the DSS of length between 1 and 40. A first observation is that the area of a preimage is always greater than its measure. A second remark is that the area $\mathcal{A}(P)$ is some $O(m(P))$, with a multiplicative constant ranging from 1 (first observation), to about 2.7 . 

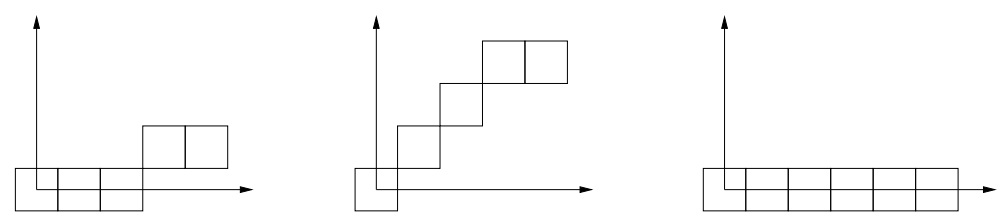

Fig. 4. Three DSS of length 5 and 6 with measure respectively 0.0738 (left) and 0.0198 (middle) and 0.099 (right).

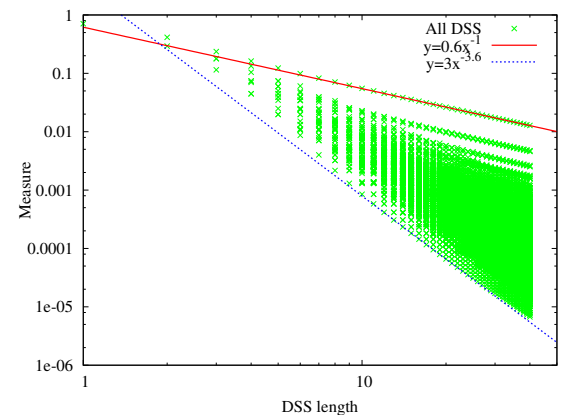

(a)

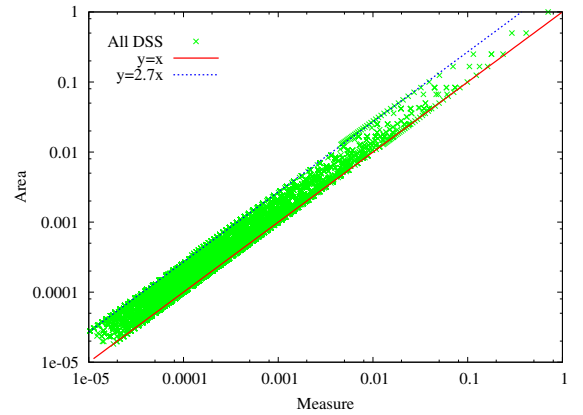

(b)

Fig. 5. Relation between the measure and other geometric features: (a) measure against DSS length in logarithmic scale, and (b) area against measure in logarithmic scale for all DSS of length between 1 and 40 .

\subsection{Measure of lines from DSS parameters}

In order to be used for contour analysis, the measure has to be computable from the output of a DSS recognition algorithm. Two main classes of DSS recognition algorithms exist: the ones that compute incrementally the preimage of the DSS (Lindenbaum and Bruckstein, 1993), and the "arithmetical" ones (DebledRennesson and Reveillès, 1995; Vialard, 1996), whose output consists in the parameters and the leaning points of the DSS. In the first case, computing the measure of a DSS is straightforward since the preimage has been computed. In the latter case however, one has to retrieve the preimage vertices before the measure computation, and this process is detailed in this section. Let us consider a DSS $S$ defined by $0 \leq p x-q y+\mu<\max (|p|,|q|), p, q, \mu \in \mathbb{Z}$. Without loss of generality, we suppose that $0 \leq p \leq q$, the other cases are symmetrical. The lower (resp. upper) leaning points of $S$ are the points satisfying $p x-q y+\mu=\max (|p|,|q|)$ (resp. $p x-q y+\mu=0$ ).

For a given DSS $S$ infinitely many parameters $(p, q, \mu)$ fulfill these properties, and in the following we only consider the strictly bounding parameters such that $S$ contains at least three leaning points. We denote by $L$ (resp. $L^{\prime}$ ) and $U$ (resp. $U^{\prime}$ ) the lower and upper leaning points of $S$ with minimal $x$-coordinate (resp. maximal $x$-coordinate). A DSS of parameters $(2,5,0)$ is depicted in Fig. 6 


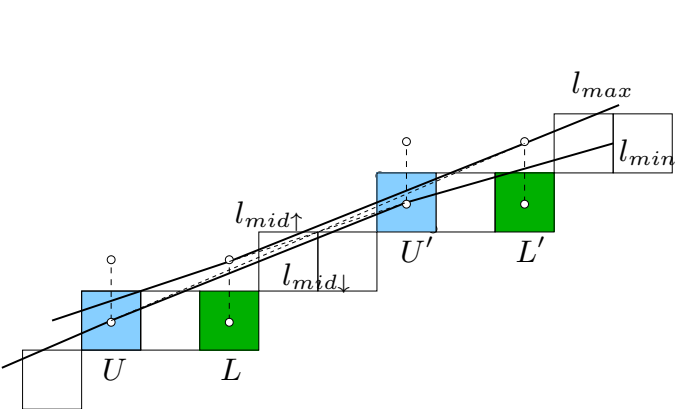

(a)

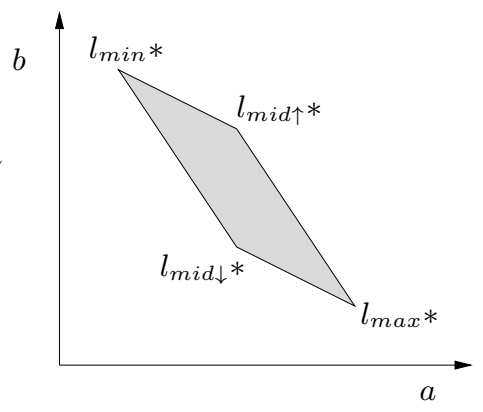

(b)

Fig. 6. Computation of the preimage vertices from the DSS parameters : in (a), a DSS $S$ with its leaning points, in (b), the preimage of this DSS.

together with its leaning points. The set of lines whose digitization contains $S$ is bounded by the straight lines $l_{\min }, l_{\max }, l_{\operatorname{mid} \downarrow}$ and $l_{m i d \uparrow}$. In the (a,b)-space, these lines map to points that are the vertices of the DSS preimage. The parameters of these lines, and thus the coordinates of the vertices of the preimage can be explicitly computed from the leaning points of $S$, as defined hereafter. The slope of a line $l$ is denoted by $a(l)$ while its intercept is denoted by $b(l)$, in reference to the (a,b)-space. Thus, in the (a,b)-space, the line $l$ maps to the point $l *$ of coordinates $(a(l), b(l))$. Moreover, the $x$-coordinate and $y$-coordinate of a point $M$ are respectively denoted by $x(M)$ and $y(M)$. We have :

\begin{tabular}{|c|c|c|c|c|}
\hline & $l_{\text {mid }}$ & $l_{m i d \uparrow}$ & $l_{\min }$ & $l_{\max }$ \\
\hline$a(l)$ & $\frac{p}{q}$ & $q$ & $\frac{y\left(U^{\prime}\right)-y(L)+1}{x\left(U^{\prime}\right)-x(L)}$ & $\frac{y\left(L^{\prime}\right)+1-y(U)}{x\left(L^{\prime}\right)-x(U)}$ \\
\hline$b(l)$ & $\frac{\mu}{q}$ & $\frac{q+1}{q}$ & $-a\left(l_{\min }\right) x\left(U^{\prime}\right)+y\left(U^{\prime}\right)$ & $-a\left(l_{\max }\right) x(U)+y(U)$ \\
\hline
\end{tabular}

This table, which is a direct consequence of the (a,b)-space and DSS definitions (see (Coeurjolly et al., 2005)), is a bridge between arithmetical DSS recognition algorithms and the measure computation. Arithmetical recognition algorithms being the fastest, this transformation is very useful for contour analysis, as we will see in Section 4.

\subsection{Representative Euclidean Straight Line of a DSS}

In applications such as the polygonal reconstruction from a digital curve, we sometimes need to associate a representative Euclidean straight line to a preimage. In the literature, such a representative straight line is usually computed as the point in the $(a, b)$-space defined as the mid-point of the preimage diagonal obtained by the two vertices of equal abscissa (Vialard, 1996). The advantage of such an approach is that both the obtained straight line and the DSS slopes are identical. 
We propose here a new definition of the representative Euclidean straight line based on integral geometry tools.

Definition 1. Given a continuous and closed domain $G(a, b)$, the representative Euclidean Straight Line is the center of mass of $G$ on the density function defined in Sect. 2.2. In other words, such a point is defined as

$$
\left(\frac{\int_{G} a d a d b}{\int_{G} d a d b}, \frac{\int_{G} b d a d b}{\int_{G} d a d b}\right)
$$

In terms of probability, the centroid definition corresponds to the expected values of the normalized probability distributions defined from the density on the $a$ and $b$ variables in the Lebesgue sense.

The denominators in Eq. (17) correspond to the measure $G$. To evaluate the numerators on a polygonal domain $P$, we can use the evaluation scheme detailed in Sect. 2.3: we decompose the integration over $P$ into a finite sum of integrations over the unit triangle. Finally, we evaluate the integrals to obtain closed formulas.

Lemma 3. The Representative Euclidean straight line of a polygonal domain $P=\left\{\left(a_{i}, b_{i}\right)\right\}_{i=0 . . n-1}$ in the $(a, b)-$ space is the point $\left(\frac{C_{a}}{m(P)}, \frac{C_{b}}{m(P)}\right)$ with

$$
\begin{aligned}
C_{a} & =\sum_{i=0}^{n-1}\left(a_{i} b_{i+1}-a_{i+1} b_{i}\right) \cdot g_{a}\left(a_{i}, a_{i+1}\right) \\
C_{b} & =\sum_{i=0}^{n-1}\left(a_{i} b_{i+1}-a_{i+1} b_{i}\right) \cdot g_{b}\left(a_{i}, a_{i+1}, b_{i}, b_{i+1}\right)
\end{aligned}
$$

and

$$
\begin{aligned}
g_{a}\left(a_{i}, a_{i+1}\right)= \begin{cases}\frac{\operatorname{arcsinh}\left(a_{i}\right)-\frac{a_{i}}{\sqrt{1+a_{i}^{2}}}}{a_{i}^{2}} & \text { if } a_{i}=a_{i+1}, \\
\frac{a_{i+1}-\operatorname{arcsinh}\left(a_{i+1}\right)}{a_{i+1}^{2}} & \text { if } a_{i}=0, \\
\frac{a_{i}-\operatorname{arcsinh}\left(a_{i}\right)}{a_{i}^{2}} & \text { if } a_{i+1}=0, \\
\frac{a_{i} \operatorname{arcsinh}\left(a_{i+1}\right)-a_{i+1} \operatorname{arcsinh}\left(a_{i}\right)}{a_{i} a_{i+1}\left(a_{i}-a_{i+1}\right)} & \text { otherwise. }\end{cases} \\
g_{b}\left(a_{i}, a_{i+1}, b_{i}, b_{i+1}\right)= \begin{cases}\frac{a_{i}\left(b_{i}+a_{i}\left(-a_{i}+\sqrt{1+\frac{1}{a_{i}^{2}}} \sqrt{1+a_{i}^{2}}\right) b_{i+1}\right)}{\sqrt{1+a_{i}^{2}}} & \text { if } a_{i}=a_{i+1}, \\
\frac{\left(b_{i}+b_{i+1}\right) \operatorname{arcsinh}\left(a_{i}\right)-\frac{a_{i+1}\left(\left(-2+\sqrt{1+a_{i+1}^{2}}\right) b_{i}+2 a_{i+1}^{3}\right)+\left(b_{i}-2 b_{i+1}\right) \operatorname{arcsinh}\left(a_{i+1}\right)}{2 a_{i+1}^{3}}}{2 a_{i+1}^{3}} a_{i}=0, \\
\frac{a_{i}\left(2 b_{i}+\left(-2+\sqrt{1+a_{i}^{2}}\right) b_{i+1}\right)+\left(-2 b_{i}+b_{i+1}\right) \operatorname{arcsinh}\left(a_{i}\right)}{2 a_{i}^{3}} & \text { if } a_{i+1}=0, \\
\text { NumApprox }\left(a_{i}, a_{i+1}, b_{i}, b_{i+1}\right) & \text { otherwise. }\end{cases}
\end{aligned}
$$

where NumApprox $\left(a_{i}, a_{i+1}, b_{i}, b_{i+1}\right)$ is the numerical approximation of

$$
\int_{0}^{1} \int_{0}^{1-b^{\prime}} \frac{\left(b_{i} a^{\prime}+b_{i+1} b^{\prime}\right) d a^{\prime} d b^{\prime}}{\left(1+\left(a_{i} a^{\prime}+a_{i+1} b^{\prime}\right)^{2}\right)^{\frac{3}{2}}}
$$


The proof of this lemma is similar to the proof of Lemma 2: $g_{a}$ and $g_{b}$ functions are obtained using a formal integration over the unit triangle. A specific point concerns the $g_{b}$ function in the general case for which no closed formula has yet been found: we failed to find a suitable parameter transformation in order to help the symbolic integrator to find a solution. However, a closed formula exists to integrate the function in Eq. (18) over a rectangular domain. Hence, the integral can be approximated using the Rectangle Approximation method (function NumApprox). Fig. 3 illustrates the center of mass for each preimage in Farey Fans of order from 0 to 5.

In Fig. 7, we compare our representative line computation to the one proposed in (Vialard, 1996) on the Farey Fan of order 4 (Fig. 7-(a)). Note that even if points in the $(a, b)$-space associated to the representative lines in both definitions may differ, the associated lines in the primal space may be visually close. Furthermore, thanks to the definition of the preimage in Eq. (16), both the restriction to positive slope straight lines $(a \in[0,1])$ and the Object Boundary Quantization (OBQ) digitization scheme makes the representative line for horizontal digital segment rotated and not perfectly horizontal (see Fig. 7-(b)). Despite, the proposed representative line definition is consistent to the straight line measure theory. Furthermore, the proposed framework can be generalized to different DSS preimage defintions, including for example, lines with negative slopes or the Gauss digitization model (see Sec. 1)

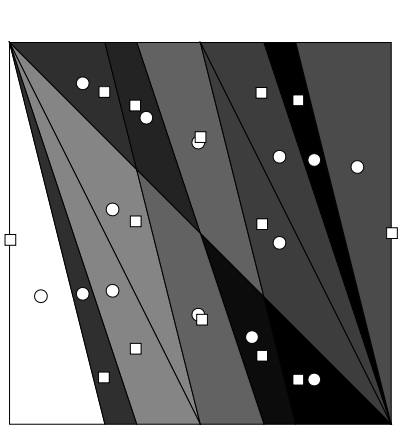

(a)

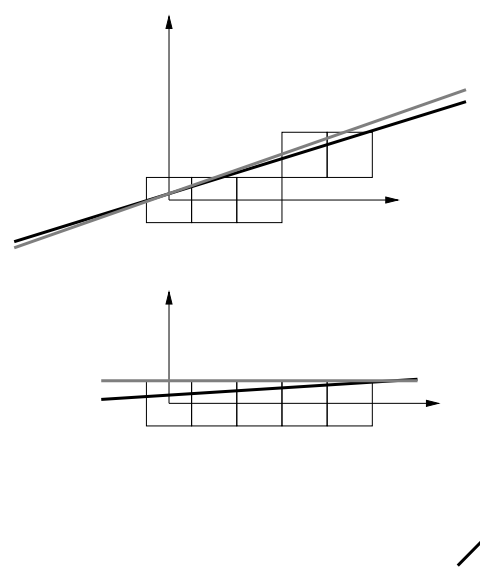

(b)

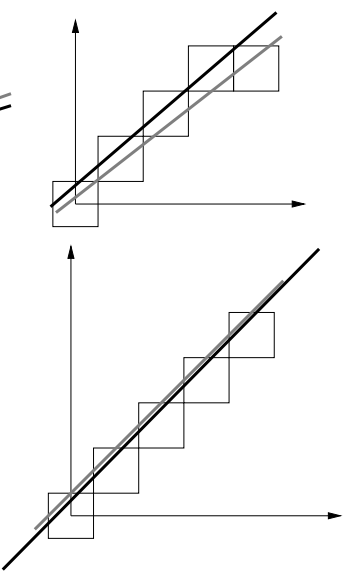

Fig. 7. Comparison between the proposed representative straight line computation (circles in $(a)$ and bold lines $(b)$ ) and the existing approach (squares in $(a)$ and gray lines in $(b))$ : (a) differences depicted in the Farey Fan of order 4 and $(b)$ in the primal space. 


\section{Contour analysis with Digital Tangent and Preimage Measures}

\subsection{Measure of DSS contained in Digital Contours}

In many shape descriptor, the analysis of DSS contained in digital contour is fundamental to obtain multigrid convergent measurements (Coeurjolly and Klette 2004; Lachaud et al. 2005, 2007). For example, at a point $p$ of a digital contour, one can estimate the tangent at $p$ as the parameters of the maximal DSS centered at $p$ (also called the digital tangent at $p$ ). Another option is to associate to each point $p$, the set of maximal digital segments containing $p$ as reviewed in (Lachaud et al., 2007). In this section, we consider the use of the measure of set of lines to have finer analysis of the DSSs contained in a contour.

As detailed above, the Farey fan of order $n$ contains all the DSS of length $n+1$. When considering the DSS belonging to digital contours, Fig. 8 details the distribution of maximal DSS measure on digital contours. As expected, only a subset of all the possible DSS of a given length $n$ are maximal DSS in digital contours. Furthermore, we can observe that the DSS with very high or very low measure are generally not maximal DSS on digital contours. The information obtained from the measure for contour analysis seems valuable compared to the length of the DSS and this point should be further investigated.

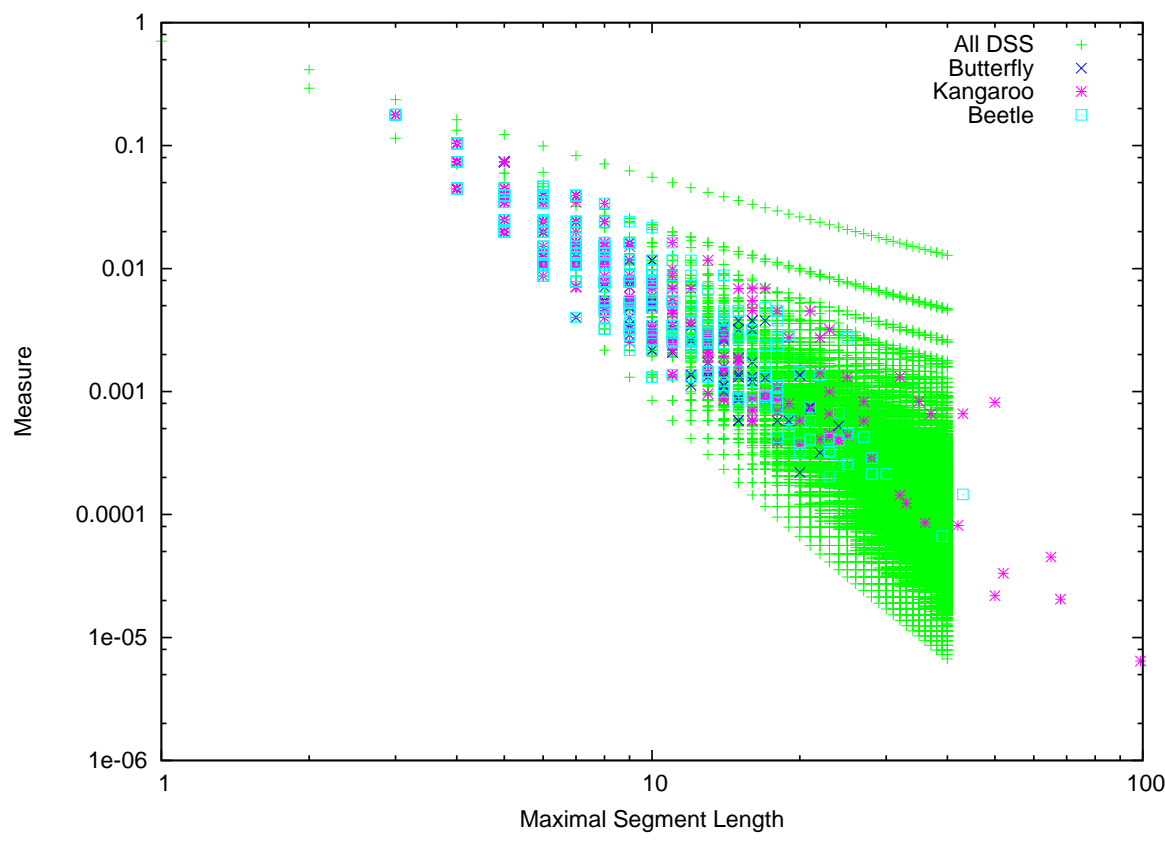

Fig. 8. Measure of maximal segment on digital contours according to maximal DSS length. 
In order to construct a shape descriptor from the measure of lines, let us first denote by $C=\left\{p_{0}, \ldots, p_{n-1}\right\}$ a digital closed curve. From the measure definition detailed above, we can design the following linearity map.

Definition 2 (Linearity maps). Given a closed contour $C$, the linearity map is a function $\lambda: C \rightarrow \mathbb{R}$ that associates for each point $p_{i} \in C$, the measure of straight lines of the digital tangent of $p_{i}$.

From this definition, if $\lambda(i)>\lambda(j)$, it means that the set of straight lines whose digitization contains the digital tangent at $p_{i}$ is wider than the set of straight lines whose digitization contains the digital tangent at $p_{j}$. Roughly speaking, the linearity map measures the uncertainty of digital tangents with respect to the straight lines. Fig. 9 illustrate the behavior of the $\lambda$ map on a digital contour. Using only the information on the set of straights lines contained in digital tangents, we can observe that preliminary results indicate that the linearity map behaves as a curvature measure: highest values corresponds small tangents and thus to high curvature parts.

\subsection{Representative Euclidean Straight Line and Digital Tangent Estimation}

In the literature, digital tangents and maximal digital segments play an important role in tangent estimation (De Vieilleville et al., 2005). Among the existing estimators, two of them are the Extended Digital Tangent and the $\lambda-M S T$ (Lachaud et al., 2005, 2007). At a point $p$, the first technique estimates the tangent as the slope of the maximal DSS obtained starting from $p$ and extending it in both direction (when a direction fails, we continue with the other one). The $\lambda$-MST is more complex, and kinds of smoothes the result considering a weighted mean of all the maximal digital segments going through a point. But it also requires to associate a slope to a DSS. Based on the DSS parameters defined in Sect. 3.1, the two previous algorithms consider the parameter $a_{0}$ as the representative slope of the considered DSS. Instead of using the value $a_{0}$, we have performed experiments in order to evaluate the impact of the use of the representative Euclidean straight line (Sect. 3.3) on the digital tangent estimation. Basically, we have used Lemma 3 to reformulate the extended tangent and $\lambda$-MST estimators available in the imagene software (Lachaud, 2003).

In Fig. 11, we illustrate a result on the extended tangent and $\lambda$-MST estimators. We have considered different analytical shapes digitized on grids with increasing resolutions (Fig. 10). For each digital contour, we estimate the mean absolute error between the estimation and the real tangent. The $y$-axis in the graphs represent the difference between the classical extended tangent estimator and the estimation using the measure based on the representative Euclidean line of the extended tangent. Hence, if this quantity is greater than 0, it means that the use of the measure enhances the quality of the estimation. In Fig. 11(a), we can see that for the pentagon, the tangent estimation is better using the results of Lemma 3. However, for the circle and the flower the results are more chaotic: using the representative Euclidean straight line even seems to generally give a 


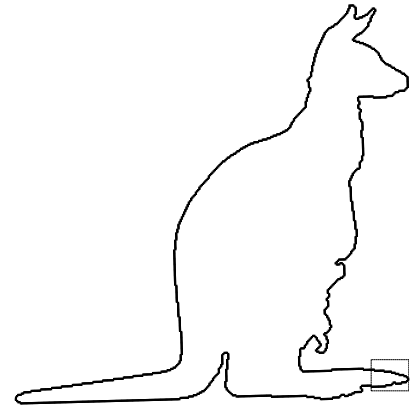

(a)

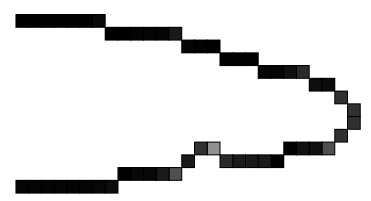

(b)

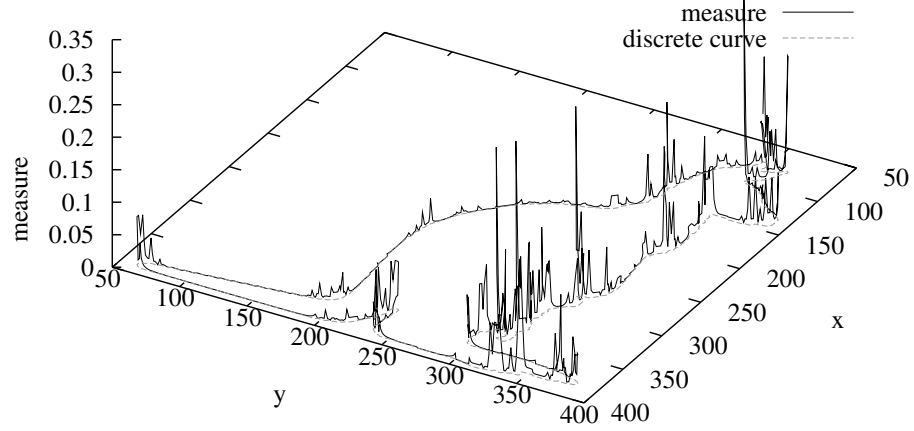

(c)

Fig. 9. Preliminary results of the linearity map for the kangaroo curve (a). Fig. (b) focuses on the kangaroo leg part: the lower the measure, the darker the pixel. In (c), we display the $\lambda$ value for each contour point. 
worse tangent estimation for the flower. Note that the standard deviations of the error lead to the same conclusion.
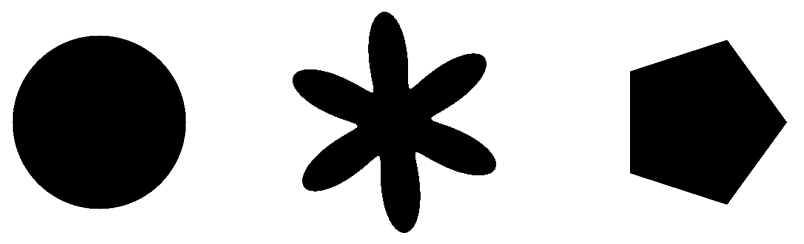

Fig. 10. Continuous shapes used in the multi-grid experiments of Fig. 11 (respectively circle,flower, rsquare and pentagon).

If we consider now the $\lambda$-MST estimator (Fig. 11(b)), first results indicate that the use of Lemma 3 in the overall estimation does not increase the quality. Further analysis are left to future works.

\section{Conclusion and Future Works}

In this paper, we have introduced concepts from integral geometry and geometric probability in the digital geometry context. More precisely, we have focused on the computation of the measure of straight lines in the $(a, b)$ parameter space which allows to first have a precise analysis of Farey Fans in terms of measure. Then, we have investigated a formal and consistent definition of a representative Euclidean straight line associated to a DSS. Finally, we have defined a new tool, so called the linearity map, to describe discrete contours. At this point, only a preliminary analysis of this feature map has been addressed. A formal comparison between such a tool and a classical curvature based descriptor is a challenging future work. Furthermore, it would be interesting to consider similar integral geometry analysis of other objects such as digital circles or digital polynomials.

Finally, the source code associated to Lemmas 2 and 3 is available at the address http://liris.cnrs.fr/david.coeurjolly/ (code, libDSSMeasure). 


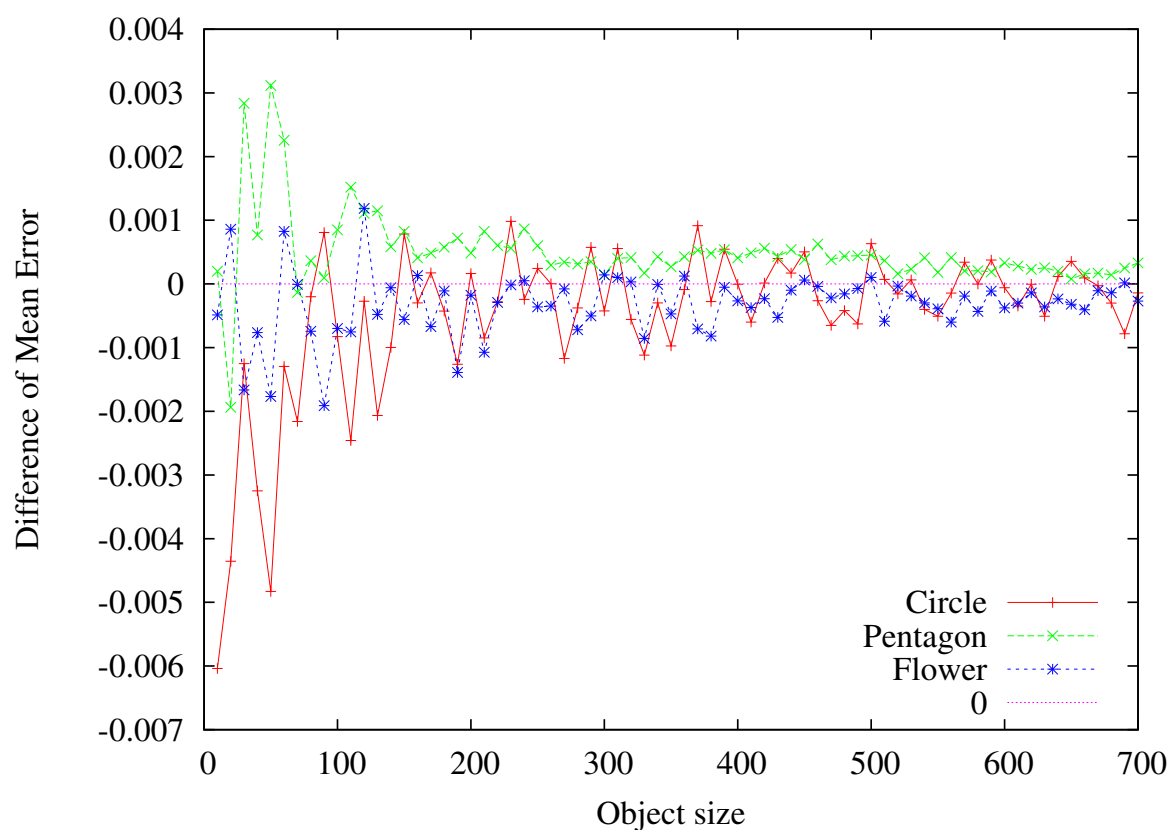

(a)

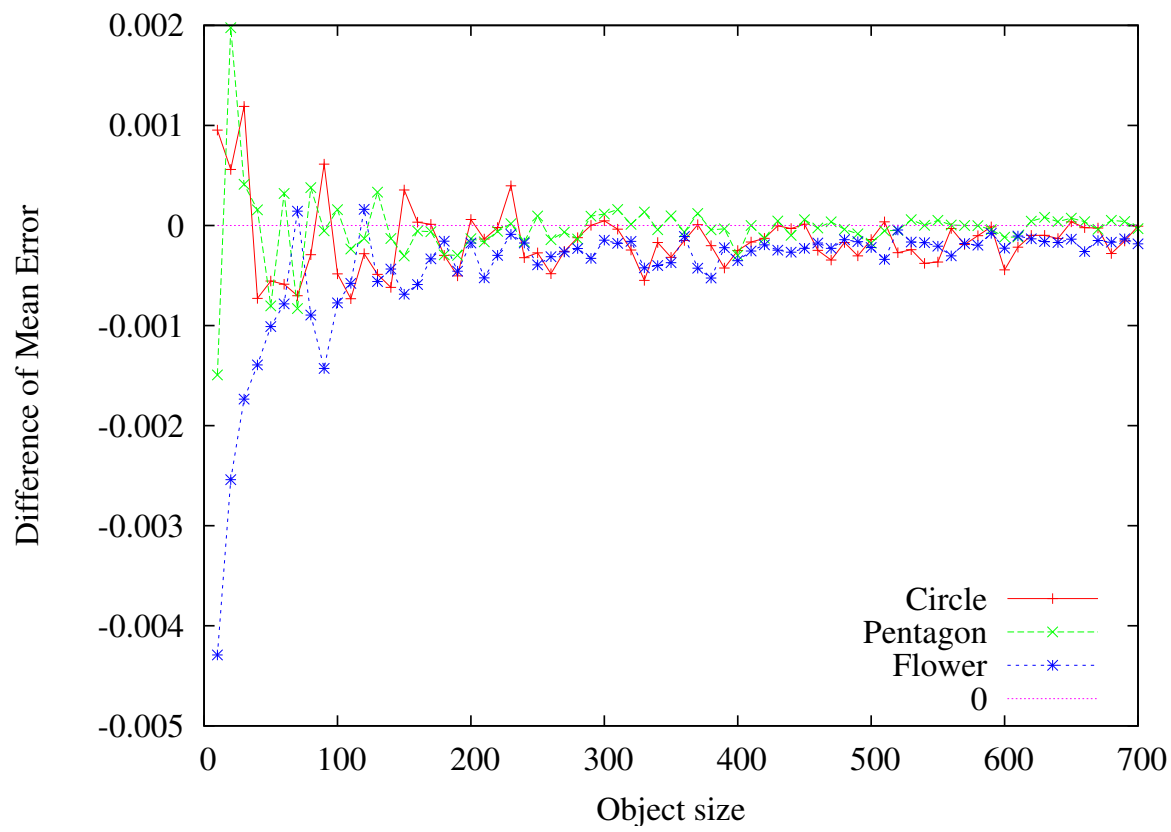

(b)

Fig. 11. Multigrid evaluation of the use of the Lemma 3 to estimate the tangent of a digital contour : (a) comparison between the classical extended tangent estimator and the use of the representative Euclidean straight line and (b) comparison between the $\lambda$-MST estimator and the $\lambda$-MST estimator tuned with the representative Euclidean straight line (see text for details). 


\section{Bibliography}

R. Breton, I. Sivignon, F. Dupont, and E. Andres. Towards an invertible euclidean reconstruction of a discrete object. In Ingela Nyström, Gabriella Sanniti di Baja, and Stina Svensson, editors, DGCI, volume 2886 of Lecture Notes in Computer Science, pages 246-256. Springer, 2003. ISBN 3-54020499-7. URL http: //springerlink . metapress. com/openurl . asp?genre= article $\backslash \&$ issn=0302-9743 \\&volume=2886 $\ \&$ spage $=246$.

V. Caglioti. On the uncertainty of straight lines in digital images. Graphical Models and Image Processing, 55(4):255-270, July 1993.

D. Coeurjolly and R. Klette. A comparative evaluation of length estimators of digital curves. IEEE Transactions on Pattern Analysis and Machine Intelligence, 26(2):252-258, feb 2004. ISSN 0162-8828.

D. Coeurjolly, I. Sivignon, F. Dupont, F. Feschet, and J. M. Chassery. On digital plane preimage structure. Discrete Applied Mathematics, 151(1-3): 78-92, 2005.

M. Crofton. On the theory of local probability. Phil. Trans. of the Royal Soc., pages 181-199, 1868.

F. De Vieilleville, Jacques-Olivier Lachaud, and F. Feschet. Maximal digital straight segments and convergence of discrete geometric estimators. In Image Analysis Proc. 14th Scandinavian Conference on Image Analysis (SCIA2005), volume 3540 of Lecture Notes in Computer Science, pages 988-1003. Springer, 2005.

I. Debled-Rennesson and J. P. Reveillès. A linear algorithm for segmentation of digital curves. In International Journal on Pattern Recognition and Artificial Intelligence, volume 9, pages 635-662, 1995.

L. Dorst and A. N. M. Smeulders. Discrete representation of straight lines. IEEE Trans. on Pattern Analysis and Machine Intelligence, 6:450-463, 1984.

L. Dorst and A. W. M. Smeulders. Decomposition of discrete curves into piecewise straight segments in linear time. In Contemporary Mathematics, volume 119, 1991.

Richard O. Duda and Peter E. Hart. Use of the hough transform to detect lines and curves in pictures. Communications of the ACM, 15(1):11-15, 1972.

A. Jonas and N. Kiryati. Digital representation schemes for 3d curves. Pattern Recognition, 30(11):1803-1816, 1997.

Nahum Kiryati, Michael Lindenbaum, and Alfred M. Bruckstein. Digital or analog hough transform? Pattern Recognition Letters, 12(5):291-297, 1991.

R. Klette and A. Rosenfeld. Digital straightness, a review. Discrete Applied Mathematics, 139(1-3):197-230, 2004a.

R. Klette and A. Rosenfeld. Digital Geometry: Geometric Methods for Digital Picture Analysis. Series in Computer Graphics and Geometric Modelin. Morgan Kaufmann, 2004b.

J.-O. Lachaud. Imagene, Generic digital Image library. http://gforge.liris.cnrs.frs/projects/imagene. 
J.-O. Lachaud. Coding cells of digital spaces: a framework to write generic digital topology algorithms. In A. Del Lungo, V. Di Gesù, and A. Kuba, editors, Proc. Int. Work. Combinatorial Image Analysis (IWCIA'2003), Palermo, Italy, volume 12 of ENDM. Elsevier, 2003.

J. O. Lachaud, A. Vialard, and F. de Vieilleville. Fast, accurate and convergent tangent estimation on digital contours. Image and Vision Computing, 25 (10):1572-1587, oct 2007. URL http://www.sciencedirect.com/science/ article/B6V09-4MBT1S8-1/2/0408c998621a93fa56ddb12589d10aaf.

Jacques-Olivier Lachaud, Anne Vialard, and François de Vieilleville. Analysis and comparative evaluation of discrete tangent estimators. In Eric Andres, Guillaume Damiand, and Pascal Lienhardt, editors, DGCI, volume 3429 of Lecture Notes in Computer Science, pages 240-251. Springer, 2005. ISBN 3-540-25513-3. URL http://springerlink.metapress.com/openurl.asp? genre $=$ article $\backslash \&$ issn $=0302-9743 \backslash \&$ volume $=3429 \backslash$ \&spage $=240$.

M. Lindenbaum and A. Bruckstein. On recursive, $o(n)$ partitioning of a digitized curve into digital straigth segments. IEEE Trans. on Pattern Analysis and Machine Intelligence, 15(9):949-953, sep 1993.

M. D. McIlroy. A note on discrete representation of lines. ATEST Technical Journal, 64(2):481-490, FEB 1985.

L. A. Santaló. Integral Geometry and Geometric Probability, volume 1 of Encyclopedia of Mathematics and Its Applications. Addison-Wesley, 1985.

J. Sklansky. Measuring concavity on a rectangular mosaic. IEEE Trans. Computer, 21(12):1355-1364, dec 1972.

A. Vialard. Geometrical parameters extraction from discrete paths. In 6th Discrete Geometry for Computer Imagery, pages 24-35. Springer-Verlag, LNCS, 1176, 1996. 